\title{
Metabolic Adaptations of Uropathogenic $E$. coli in the Urinary Tract
}

\author{
Riti Mann, Daniel G. Mediati, Iain G. Duggin, Elizabeth J. Harry and Amy L. Bottomley* \\ Faculty of Science, The iThree Institute, University of Technology Sydney, Ultimo, NSW, Australia
}

Escherichia coli ordinarily resides in the lower gastrointestinal tract in humans, but some strains, known as Uropathogenic E. coli (UPEC), are also adapted to the relatively harsh environment of the urinary tract. Infections of the urine, bladder and kidneys by UPEC may lead to potentially fatal bloodstream infections. To survive this range of conditions, UPEC strains must have broad and flexible metabolic capabilities and efficiently utilize scarce essential nutrients. Whole-organism (or "omics") methods have recently provided significant advances in our understanding of the importance of metabolic adaptation in the success of UPECs. Here we describe the nutritional and metabolic requirements for UPEC infection in these environments, and focus on particular metabolic responses and adaptations of UPEC that appear to be essential for survival in the urinary tract.

\section{OPEN ACCESS}

Edited by:

Klaus Heuner,

Robert Koch-Institute, Germany

Reviewed by:

Charles Martin Dozois,

Institut national de la recherche

scientifique, Canada

Sheryl S. Justice,

The Ohio State University,

United States

*Correspondence:

Amy L. Bottomley

amy.bottomley@uts.edu.au

Received: 31 March 2017

Accepted: 24 May 2017

Published: 08 June 2017

Citation:

Mann R, Mediati DG, Duggin IG,

Harry EJ and Bottomley AL (2017)

Metabolic Adaptations of

Uropathogenic E. coli in the

Urinary Tract.

Front. Cell. Infect. Microbiol. 7:241.

doi: 10.3389/fcimb.2017.00241
Keywords: uropathogenic E. coli, UPEC, urinary tract infections, metabolism, metabolomics, virulence

\section{INTRODUCTION}

Urinary tract infections (UTIs) are one of the most common bacterial infections worldwide, accounting for $\sim 150$ million cases annually (Stamm and Norrby, 2001). This places a significant financial burden on the health-care system, costing up to USD\$6 billion on treatment and management each year. UTIs can be community-acquired or health-care related, and occurs in both men and women. Women are significantly more at risk with almost $50 \%$ of all women experiencing a UTI at least once in their lifetime (Foxman, 2003; Brumbaugh et al., 2013).

UTIs are categorized as complicated or uncomplicated on the basis of the type and duration of antimicrobial therapy given to the patient (Hooton, 2012; Flores-Mireles et al., 2015). Uncomplicated UTIs typically affect children, women and the elderly who are otherwise healthy and have no structural urinary tract abnormalities. These are differentiated into upper and lower UTIs, most commonly pyelonephritis (kidney) and cystitis (bladder), respectively. Uncomplicated UTIs rarely cause any serious damage, and can be naturally cleared by the host immune response even without antibiotic therapy (Hooton, 2012). Complicated UTIs on the other hand require a prolonged therapy and are associated with an increased risk of chronic and/or recurrent infection. Complicated UTIs are associated with urinary tract abnormalities such as urinary obstruction and retention, immunosuppression, previous antibiotic exposure, and renal failure. Together these factors compromise the urinary tract and increase the risk of serious complications and treatment failure (Lichtenberger and Hooton, 2008).

A range of pathogens have been implicated in UTIs, including Gram-positive and Gramnegative bacteria such as Escherichia coli, Proteus mirabilis, Klebsiella pneumoniae, Staphylococcus saprophyticus, and Enterococcus faecalis. However, the disease is predominantly caused by Uropathogenic E. coli (UPEC; Schulz, 2011; Flores-Mireles et al., 2015; Su et al., 2016b), which 
accounts for up to 75\% of all cases (Foxman, 2002, 2003; Martinez and Hultgren, 2002) and $95 \%$ of community-acquired cases (Kucheria et al., 2005).

UPEC are a pathotype of extraintestinal pathogenic E. coli (ExPEC) and originate from the intestinal microbiome. Within the intestine, UPEC rarely cause any complications and exist in a beneficial symbiotic relationship with intestinal microflora (Wiles et al., 2008). However, UPEC have adapted the ability to disseminate and colonize other host environments such as the urinary tract and bloodstream. Virulence factors, such as toxins, modify, and damage the host to promote infection (FloresMireles et al., 2015). In addition, physiological factors that do not directly damage the host but nevertheless are essential for UPEC growth and survival in the urinary tract are now being appreciated for their role in pathogenesis (Alteri and Mobley, 2012). The capacity of UPEC to utilize nutritionally-diverse environments such as the intestines, urine, bladder, kidneys, and bloodstream clearly plays a significant role in its pathogenesis (Brown et al., 2008; Eisenreich et al., 2010); UPEC metabolism is tightly regulated and highly responsive to nutrient availability, allowing survival with a wide range of nutrients in competitive, fluctuating environments.

This review will discuss the nutritional and metabolic responses of UPEC as it moves from the intestine to the harsh environment of the urinary tract, where virulent infection starts. Nutrients available for UPEC growth in the intestine and urinary tract have been compared in order to explore these two environments as growth mediums for UPEC. Genetic and metabolic responses that allow UPECs to survive in and cause infection in the urinary tract are discussed in relation to the nutrients available inside the urinary tract. As the role of metabolism during UPEC infection is critical to advance our understanding of pathogenesis, we reflect on the prospect of UPEC metabolism as a potential drug target, to combat the antibiotic resistance that has already developed against $70 \%$ of the drugs currently being used clinically for UPEC-induced UTIs (Foxman, 2002).

\section{UPEC LIFESTYLE FROM INTESTINE TO THE URINARY TRACT}

The gastrointestinal tract is considered the primary reservoir of UPEC in humans. For successful colonization in their main habitat, the colon, E. coli first needs to survive passage through the acidic conditions of the stomach, and upper intestine, and then penetrate the viscous upper mucus layer of the colon epithelium (Møller et al., 2003), survive other host defense mechanisms (Bergstrom et al., 2012) and compete with other strains and species of the complex intestinal surface microbiota for acquisition and utilization of nutrients (Freter et al., 1983; Rang et al., 1999; Miranda et al., 2004). Some E. coli cells remain or are shed into the intestinal lumen and then excreted in feces.

UTIs are usually initiated when UPEC contaminate and colonize the urethra and migrate into the bladder lumen. Most of the characterized strains of UPEC invade the bladder epithelium and undergo an intracellular infection cycle (Martinez et al., 2000; Justice et al., 2004) and there is evidence that this occurs in most human UTIs (Rosen et al., 2007). The infection cycle is a complex pathway involving epithelial attachment, invasion of host cells, and intracellular proliferation, leading to the eventual rupture of the bladder epithelial cell, dissemination and reinfection of surrounding epithelial cells (Justice et al., 2004, 2006a; Andersen et al., 2012). Infections of the lower urinary tract have the potential to progress to the kidneys and enter the bloodstream causing potentially fatal urosepsis, as shown in Figure 1 (FloresMireles et al., 2015).

Many studies have focused on virulence factors that play a role in mediating UPEC colonization of the bladder, most notably the initial attachment of bacterial adhesive structures to the luminal surface of the bladder epithelium. This step of infection has been quite well characterized and has been a focus of attention in the development of therapies for UTIs (Langermann et al., 2000; Asadi et al., 2014). UPEC surface fibers, in particular Type 1 pili and their tip adhesin $(\mathrm{FimH})$, recognize mannosylated glycoprotein receptors present on the host epithelium, mediating UPEC binding (Jones et al., 1995; Martinez et al., 2000). UPEC also express a variety of other proteins on their surface, which facilitates bacterial attachment (see Mulvey, 2002). Once bound, UPEC may become internalized via endocytosis (Mulvey et al., 1998); activation of the host Rho-family proteins, such as RhoA, Cdc42, and Rac1 result in the rearrangement of the host cell membrane, engulfing the bacteria in endocytic vesicles (Martinez et al., 2000; Martinez and Hultgren, 2002).

Within the intracellular environment, which offers a degree of protection from innate immune responses, UPEC grow as dense clusters of bacteria encased in a polysacchariderich matrix (Anderson et al., 2003). These clusters, known as intracellular bacterial communities (IBCs), have been observed in the murine model and the human cell-culture model of UTI, as well as shed into human urine (Anderson et al., 2003; Justice et al., 2004; Rosen et al., 2007; Andersen et al., 2012). As UPEC proliferate and the cluster develops to overwhelm the host cell, subpopulations of differentiated bacteria develop, including highly motile bacteria and extensive bacterial filaments-cells that have continued growth with an arrest of cell division (Justice et al., 2004, 2006b; Andersen et al., 2012). The development of motile and filamentous bacteria accompanies host cell rupture, and promotes dissemination, adhesion and colonization of surrounding host surfaces, whilst helping avoid consumption by the innate immune system (Justice et al., 2006b; Andersen et al., 2012). UPEC filaments have the potential to divide and reform to the regular rodshaped cells that are able to invade new host cells (Rosen et al., 2007; Andersen et al., 2012). During these changes, UPEC would experience rapid substantial changes in nutritional status.

\section{NUTRITIONAL ASPECTS OF THE INTESTINE AND URINARY TRACT ENVIRONMENTS FOR UPEC}

UPEC must rapidly adapt to new environments as they transition between the intestinal lumen and urinary tract, in both intracellular and extracellular host microenvironments. In 


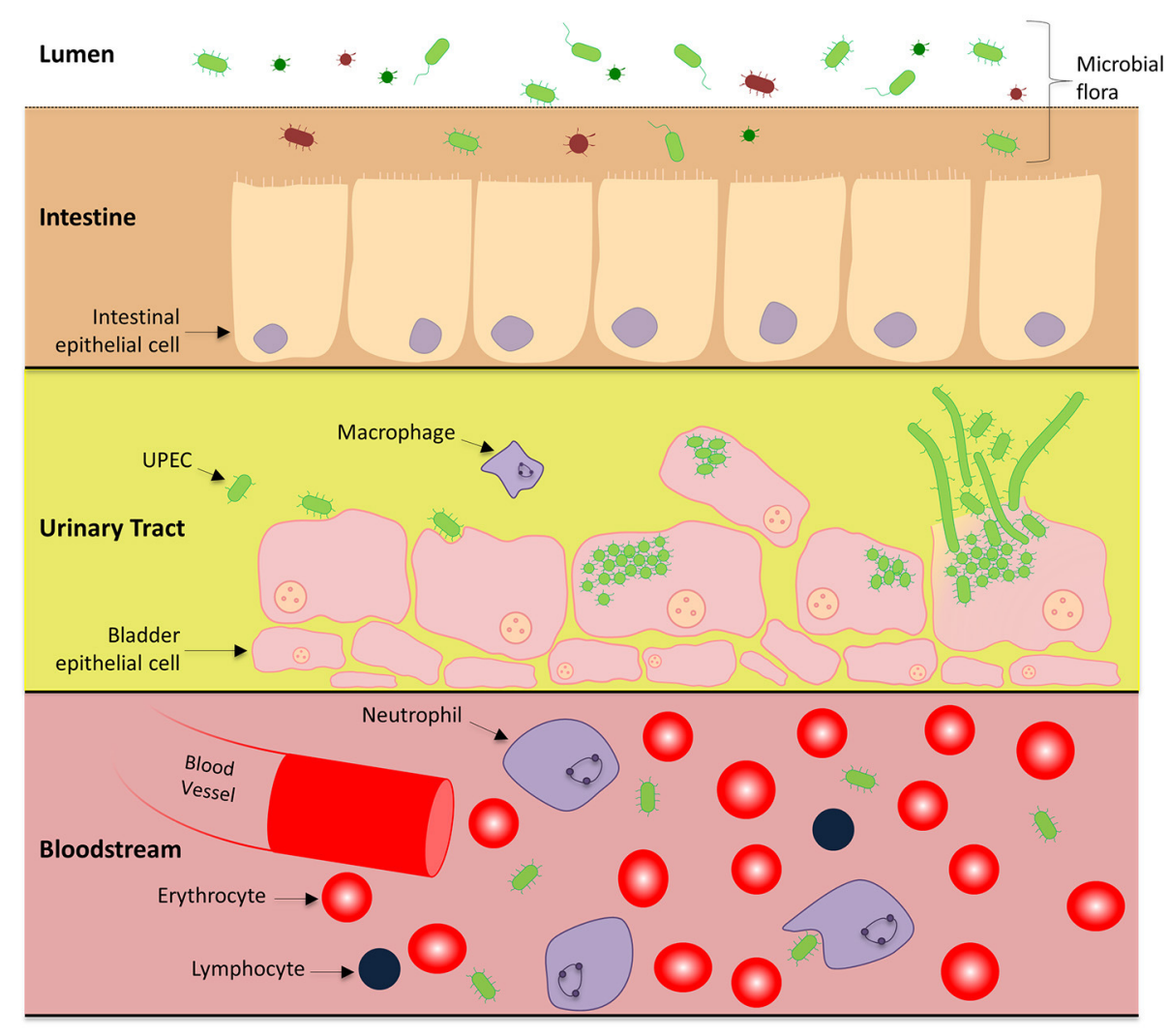

FIGURE 1 | UPEC pathogenesis across multiple microenvironments: Uropathogenic E. coli harmlessly grow in the human intestines as part of the microbiome. Within this environment, UPEC interact with the intestinal epithelial cells in a symbiotic relationship, however there is competition for nutrients between other microorganisms. UPEC has also adapted the ability to cause urinary tract infections and urosepsis by transitioning to a pathogenic lifecycle in the urinary tract and bloodstream. To gain a stronghold within the urinary tract, UPEC express numerous pili systems to facilitate attachment to the superficial bladder epithelial cell layer. Invasion into the host cell initiates replication immediately to form IBCs and a subpopulation undergoes cell elongation (filamentation). Eventually the epithelial cell is overloaded and UPEC escape, rupturing open the host cell releasing motile short and elongated cells which can infect neighboring host epithelia to continue the infective cycle.

the urinary tract, urine is one of the major sources of nutrients encountered by UPEC during the extracellular phase of the infection cycle. Table 1 outlines the principle compounds that demonstrate the potential to serve as sources of essential elements within the intestine and urine that can support UPEC growth.

\section{Intestine}

Within the intestine, most UPEC strains are thought to exist like other strains of commensal E. coli, as they do not directly cause pathogenic infection there. The major source of nutrients utilized by $E$. coli is provided by the mucus layer in which they live, which contains surface-associated and secreted glycoproteins (mucins) that are catabolized by the intestinal microbiota to yield a variety of sugars, including $\mathrm{N}$-acetylneuraminic acid and $\mathrm{N}$-acetylglucosamine that UPEC can utilize as a source of carbon and energy (Severi et al., 2007; Katouli, 2010). Proteins, lipids and nucleic acids present in the intestinal mucus layer, including those originating from shed host epithelial cells and exogenous dietary fiber may also be utilized as a nutrient source (Conway et al., 2004). Degraded components of mucus and the epithelium may also diffuse into the intestinal lumen, where they can be utilized during UPEC metabolism within the intestine. The competition for carbon sources, particularly sugars, in the complex ecosystem of the colon is considered the main nutritional limitation for E. coli in this environment (Conway and Cohen, 2015). Consequently, E. coli have developed the capacity to simultaneously metabolize numerous types of sugars, and independently and rapidly regulate their production of the enzymes required, in order to compete (Maltby et al., 2013). Essential salts and metal ions are present in sufficient quantities, however, some essential ions, particularly iron and zinc, may be limiting and subject to intense competition (Gielda and DiRita, 2012; Kortman et al., 2014).

\section{Urine}

The urinary tract offers a very different and diverse set of microenvironments that may be utilized by UPEC if the numerous nutritional and immunological stresses encountered here can be overcome. UPEC nutrition and metabolic responses during the invasive intracellular infection phases are described further below. Here, we outline the constituents of urine in relation to UPEC nutrition, since survival or growth in urine is 
TABLE 1 | Potential sources of essential elements in two of the principal environments supporting UPEC growth, the upper mucus layer in the colon, and urine in the urinary tract, with concentrations in indicated where known (C-carbon, O-oxygen, H-hydrogen, N-nitrogen, S-sulfur, P-phosphorus, K-potassium, Mg-magnesium, Ca-calcium, Fe-iron, Na-sodium, Cl-chlorine).

\begin{tabular}{|c|c|c|c|c|c|}
\hline \multicolumn{2}{|l|}{ Intestine } & \multicolumn{4}{|c|}{ Urine } \\
\hline Nutritional source & $\begin{array}{l}\text { Elementary } \\
\text { requirement } \\
\text { of UPEC fulfilled }\end{array}$ & Nutritional source & $\begin{array}{l}\text { Concentration } \\
(\text { Mean } \pm S D)^{\star}\end{array}$ & References & $\begin{array}{l}\text { Elementary } \\
\text { requirement } \\
\text { of UPEC fulfilled }\end{array}$ \\
\hline Polysaccharides & $\mathrm{C}, \mathrm{O}, \mathrm{H}$ & Urea & $22.5 \pm 4.4 \mathrm{mM}$ & Bouatra et al., 2013 & $\mathrm{C}, \mathrm{N}, \mathrm{O}, \mathrm{H}$ \\
\hline Dietary fibers & $\mathrm{C}, \mathrm{O}, \mathrm{H}$ & Creatinine & $10.4 \pm 2.0 \mathrm{mM}$ & Bouatra et al., 2013 & $\mathrm{C}, \mathrm{N}, \mathrm{O}, \mathrm{H}$ \\
\hline $\begin{array}{l}\text { Glycoproteins (N-acetylneuraminic } \\
\text { acid, N-acetylglucosamine) }\end{array}$ & $\mathrm{C}, \mathrm{N}, \mathrm{O}, \mathrm{H}$ & Hippuric acid & $298.5 \pm 276.8 \mu \mathrm{M}$ & Bouatra et al., 2013 & $\mathrm{C}, \mathrm{N}, \mathrm{O}, \mathrm{H}$ \\
\hline Glycolipids & $\mathrm{C}, \mathrm{O}, \mathrm{H}$ & Organic acids: Citric acid & $280.6 \pm 115.2 \mu \mathrm{M}$ & Bouatra et al., 2013 & $\mathrm{C}, \mathrm{O}, \mathrm{H}$ \\
\hline Proteins & $\mathrm{C}, \mathrm{N}, \mathrm{O}, \mathrm{H}, \mathrm{S}$ & Amino acids: D-serine & $3.0-40 \mu \mathrm{g} / \mathrm{mL}$ & $\begin{array}{l}\text { Cosloy and McFall, 1973; } \\
\text { Brückner et al., 1994; } \\
\text { Pätzold et al., } 2005\end{array}$ & $\mathrm{C}, \mathrm{N}, \mathrm{O}, \mathrm{H}$ \\
\hline Lipids & $\mathrm{C}, \mathrm{O}, \mathrm{H}$ & Nucleic acids & - & Bouatra et al., 2013 & $\mathrm{C}, \mathrm{N}, \mathrm{O}, \mathrm{H}, \mathrm{P}$ \\
\hline Nucleic acids & $\mathrm{C}, \mathrm{N}, \mathrm{O}, \mathrm{H}, \mathrm{P}$ & Oxytocin & $0.9 \pm 0.1 \mathrm{pM}$ & Bouatra et al., 2013 & $\mathrm{C}, \mathrm{N}, \mathrm{O}, \mathrm{H}, \mathrm{S}$ \\
\hline Phospholipids & $\mathrm{C}, \mathrm{O}, \mathrm{H}, \mathrm{P}$ & Angiotensin II & $1.2 \pm 0.2 \mathrm{pM}$ & Bouatra et al., 2013 & $\mathrm{C}, \mathrm{N}, \mathrm{O}, \mathrm{H}$ \\
\hline Ribose & $\mathrm{C}, \mathrm{O}, \mathrm{H}$ & Melatonin & $3.3 \pm 2.7 \mathrm{pM}$ & Bouatra et al., 2013 & $\mathrm{C}, \mathrm{N}, \mathrm{O}, \mathrm{H}$ \\
\hline Fucose & $\mathrm{C}, \mathrm{O}, \mathrm{H}$ & Ammonia & $2.8 \pm 0.9 \mathrm{mM}$ & Bouatra et al., 2013 & $\mathrm{~N}, \mathrm{H}$ \\
\hline Mannose & $\mathrm{C}, \mathrm{O}, \mathrm{H}$ & Sodium & $14.7 \pm 9 \mathrm{mM}$ & Bouatra et al., 2013 & $\mathrm{Na}$ \\
\hline Gluconate & $\mathrm{C}, \mathrm{O}, \mathrm{H}$ & Chlorine & $8.8 \pm 6.2 \mathrm{mM}$ & Bouatra et al., 2013 & $\mathrm{Cl}$ \\
\hline Other dietary elements & $\mathrm{K}, \mathrm{Mg}, \mathrm{Ca}, \mathrm{Fe}$ & Potassium & $4.6 \pm 0.1 \mathrm{mM}$ & Bouatra et al., 2013 & K \\
\hline
\end{tabular}

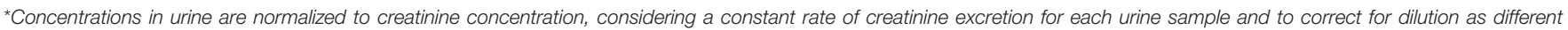
volumes of urine samples were taken for analysis (Bouatra et al., 2013).

common to UPEC's successful transition between the multiple surface and sub-surface niches throughout the urinary system.

Understanding the complete metabolic profile of urine that can be utilized by UPEC is challenging mainly due to urines great compositional complexity and variability. Nonetheless, advances in techniques such as metabolomics allow large-scale simultaneous analysis of compounds in complex biological mixtures and have greatly expanded knowledge of the metabolic profile of urine (Patti et al., 2012).

\section{The Human Urine Metabolome Database}

All metabolites identified in pooled human urine to date are available in a database termed the Urine Metabolome Database (UMDB; Bouatra et al., 2013). Currently it contains 2,651 metabolites, with 220 metabolites specifically associated with different conditions of disease. Although, the human urine metabolome shows some similarity to the human serum metabolome (Psychogios et al., 2011), there are more than 484 compounds uniquely identified in urine (Bouatra et al., 2013). Whilst this indicates that there may be a subset of metabolites that are unique to urine, it is likely that the composition of urine is similar to blood, although certain metabolites from the blood are most likely concentrated by the kidneys, resulting in them being present at much higher concentrations compared to serum, and thus above the detection levels for current metabolome analysis (Bouatra et al., 2013).

Urine contains relatively high concentrations of urea, creatinine, amino acids, organic acids, inorganic ions such as ammonia, sodium and potassium, purines, pyrimidines, and many water-soluble toxins, which must be either utilized or tolerated by UPEC (Table 1; Vejborg et al., 2012; Bouatra et al., 2013). In fact, urine contains 5-10 times more compounds and exhibits 2-3 times more chemical diversity as compared to other biological fluids like saliva (Takeda et al., 2009) and cerebral spinal fluid (Mandal et al., 2012). The major metabolites of urine are amino acids and carbohydrates. D-serine is the most abundant amino acid in mammalian urine present at concentrations between 30 and $379 \mu \mathrm{Mol} / \mathrm{L}$ (Brückner et al., 1994; Pätzold et al., 2005), whilst organic metabolites such as hydroxyl acid, citric acid, ammonia, creatinine, and hippuric acid are also present in significant quantities (Table 1). Despite containing high concentrations of many metabolites that may be utilized by UPEC, urine is classified as a fairly nutrient-limited growth medium, because arginine, methionine, valine, uracil, adenine and isoleucine are in limited supplies (Vejborg et al., 2012), and iron is present in limited concentrations (Stamey and Mihara, 1980), similar to many host environments harboring bacteria. UPEC would largely rely on its capacity for de novo synthesis of these and many other essential metabolites. The least abundant metabolites available to UPEC in urine include sugars, particularly glucose, specific peptides and hormones such as oxytocin, angiotensin, and melatonin (Bouatra et al., 2013); however despite being present in such low concentrations these components do have the potential to fulfill the elementary requirements of UPEC when catabolized. Thus, whilst urine is perhaps nutritionally more challenging to support bacterial growth compared to the intestinal environment, uropathogens, including most UPEC strains, have well-developed metabolic capacity to survive or thrive in this and other environments of the urinary tract. 


\section{GENETIC AND METABOLIC RESPONSES IN UPEC PATHOGENESIS}

The consequence of the metabolic diversity of urine on the growth of UPEC within the urinary tract still remains to be fully understood. Considering the different nutritional standings of the intestine and urinary tract and the variability within each, urinary metabolites are most likely to activate genetic and metabolic adaptive strategies in UPEC in order to survive and cause infection inside the urinary tract, some of which are detailed here.

\section{Central Carbon Metabolism}

During intracellular UPEC colonization of the bladder and kidneys, aerobic respiration and carbon metabolism are essential for survival (Figure 2; Alteri et al., 2009). UPEC pyelonephritis isolate CFT073 defective in $s d h B$, the succinate dehydrogenase enzyme involved in the conversion of fumarate to succinate during the tricarboxylic acid cycle, is significantly outcompeted by the isogenic parental strain in mouse bladder and kidney UTI models (Alteri et al., 2009). Similarly, disruption of gluconeogenesis by deletion of $p c k A$, encoding for phosphoenolpyruvate carboxykinase which catabolizes oxaloacetate to phosphoenol pyruvate, results in a significant reduction in fitness to colonize both bladder and kidneys in vivo when compared to wild-type (Alteri et al., 2009). However, mutants defective in gluconate catabolism of the Entner-Doudoroff pathway, by deletion of edd, do not affect in vivo fitness in a mouse UTI model, suggesting this pathway is dispensable in UPEC pathogenesis of UTIs (Alteri et al., 2009), and indicates a specific metabolic adaptation by UPEC during bladder infection that utilizes gluconeogenesis and the tricarboxylic acid cycle. It should be noted that the infection model used in this study only demonstrates the fitness defect in the mutants of these enzymes as compared to wild type and does not rule out the importance of other metabolic adaptations during UPEC infection. Interestingly, in a kidney mouse model of UTI, deletion of tpiA, the triosephosphate isomerase involved in glycolysis, was found to have attenuation and a colonization defect in the mouse kidneys, but not in the bladder (Alteri et al., 2009). Thus, it appears that the glycolysis pathway is dispensable during the initial establishment of infection within the bladder, but is critical for colonization of the kidneys. This evidence suggests that gluconeogenesis and the tricarboxylic acid cycle must come to completion for UPEC pathogenesis during intracellular infection of the bladder, whilst glycolysis is important in kidney colonization during UTIs.

$\beta$-galactosides and sorbitol appear to provide a major source of carbon during intracellular infection in the urinary tract as

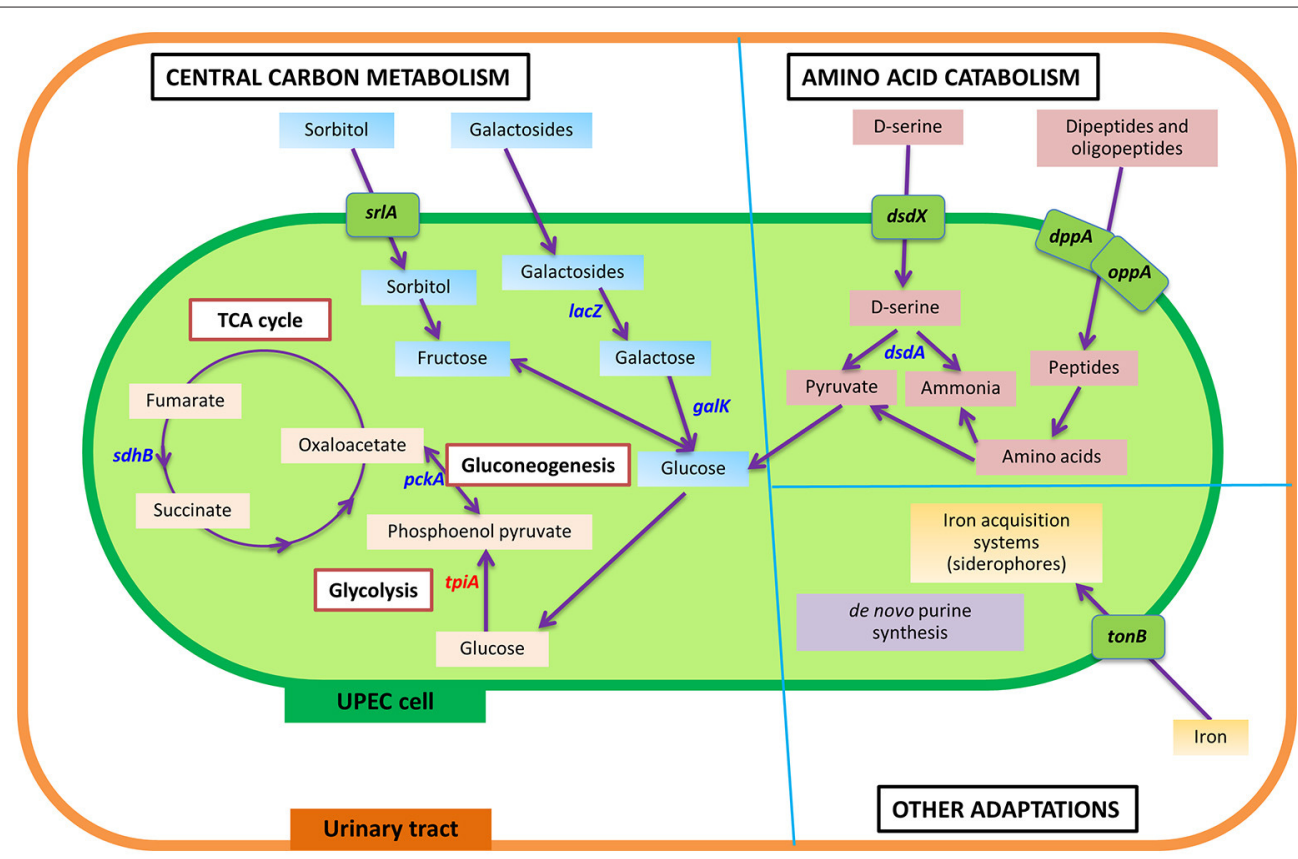

FIGURE 2 | Summarized view of the main metabolic responses of UPEC during urinary tract infection, as detailed in text under the section "Genetic and metabolic responses in UPEC pathogenesis." In comparison to the intestine, wherein glycolysis and Entner-Doudoroff pathways are shown to be important for UPEC survival, UPEC in the urinary tract displays a number of metabolic adaptations in central carbon metabolism, amino catabolism and other pathways, to cause infection in the urinary tract. Genes identified to play a role in UPEC pathogenesis are shown, having detailed information in text. Genes in blue color denote genes that play a role in UPEC fitness in the urinary tract, or result in attenuation in a mouse or in vitro model of invasion and intracellular bacterial community formation. The gene in red denotes a gene specifically identified to play a role in kidney infection. Transporters are shown in green boxes over the cell membrane. sdhB, succinate dehydrogenase; $p c k A$, phosphoenolpyruvate carboxykinase; tpiA, triosephosphate isomerase; srlA, sorbitol transporter; lac Z, $\beta$-galactosidase; galK, galactokinase; $d s d X$, D-serine specific transporter; $d s d A$, D-serine deaminase; $d p p A$, periplasmic dipeptide transport protein; oppA, periplasmic oligopeptide-binding protein; tonB, ferric iron uptake mediator; pur, genes involved in purine synthesis. 
both the sorbitol transporter, encoded by $\operatorname{srl} A$, and the galactoside catabolism gene, lac $Z$ (encoding $\beta$-galactosidase that cleaves the glycosidic bond in galactosides to release galactose), were found to be significantly upregulated during bladder cell colonization (Conover et al., 2016). This is further supported by galK mutants (the gene product being involved in conversion of galactose to glucose) producing significantly smaller IBCs compared to the wild-type in a mouse UTI model, with both mutants eventually being out-competed after 2 weeks of intracellular infection (Conover et al., 2016). Positive X-Gal (5-bromo-4-chloro-3indolyl- $\beta$-D-galactopyranoside) staining of UPEC during the IBC stage of a mouse UTI model also implies strong $\beta$ galactosidase activity (Justice et al., 2006b). Together, evidence suggests glucose availability within intracellular infection is limiting and UPEC are adapted to utilize galactose and sorbitol as their prime carbon sources. This is in accordance with urine metabolome studies, which show that glucose is rarely abundant in the urinary tract unless in a diabetic situation (Bouatra et al., 2013; Mobley, 2016).

Whilst glycolysis appears to have a critical role in the colonization of kidneys by UPEC, the importance of this metabolic pathway is not restricted to this E. coli pathotype or host environment. Both glycolysis and the Entner-Doudoroff pathway are vital for the survival of commensal E. coli in the mouse intestine as the deletion of pgi (phosphoglucose isomerase) and edd (6-phosphogluconate dehydratase) genes, having major roles within glycolysis and the EntnerDoudoroff pathway respectively, each display attenuated intestinal colonization in a competitive murine colonization model (Sweeney et al., 1996; Chang et al., 2004). Conversely, whilst gluconeogenesis and the tricarboxylic acid cycle are dispensable during UPEC intestine colonization (Chang et al., 2004), they are critical for UPEC survival during bladder infection, demonstrating the metabolic flexibility of UPEC to adapt to nutrient availability within diverse host environments.

\section{Amino Acid Catabolism}

The ability of UPEC to exploit the high mixtures of amino acids and complex small peptides present within human urine has long been considered an adaptation to this environment. The utilization of these resources by UPEC is supported by the upregulation of genes involved in amino acid catabolism during growth in human urine, such as $d s d A$ (D-serine deaminase) (Snyder et al., 2004), and microarray studies have demonstrated the upregulation of arginine, serine and histidine transporters in UPEC during urine growth when compared to LuriaBertani media (Conover et al., 2016). Both serine and arginine auxotrophs do not exhibit any major fitness defects in a mouse UTI model (Alteri et al., 2009), indicating that these amino acids are effectively utilized from the growth milieu. Small peptide uptake is also important for UPEC infection, as $d p p A$ (periplasmic dipeptide transport protein) and oppA (periplasmic oligopeptide-binding protein) deletion mutants defective in peptide transport in CFT073, were significantly out-competed by wild-type in the mouse bladder model of UTI (Alteri et al., 2009). Interestingly, a subset of genes involved in amino acid utilization and synthesis, most notably tryptophan and cysteine, were downregulated during the IBC stage of infection (Conover et al., 2016), and strains that are auxotropic for these amino acids have the ability grow to a high density in urine (Hull and Hull, 1997). This suggests that short peptide and selective amino acid uptake and catabolism is necessary for growth in human urine and correlates with the high concentrations of amino acids reported in the metabolomic analysis of human urine (Table 1). However, small peptides and amino acids must be available from alternate sources apart from urine during the infection cycle, as UPEC mutants auxotrophic in arginine, glutamine and guanine synthesis have all shown a severe growth defect in human urine, while leucine, methionine and phenylalanine auxotrophs displayed a moderate growth rate (Hull and Hull, 1997).

A potential metabolic signaling mechanism evident in UPEC during urine growth is that of the amino acid D-serine, present in high concentration $(3.0-40 \mu \mathrm{g} / \mathrm{mL}$ ) in human urine (Cosloy and McFall, 1973; Brückner et al., 1994; Pätzold et al., 2005). Whilst potentially bacteriostatic at these concentrations, the expression of D-serine deaminase in UPEC isolates allows for catabolism of this metabolite (Roesch et al., 2003; Snyder et al., 2004). Because of this, catabolism of D-serine was suggested to be a vital signaling mechanism for virulence factor expression as deletion of $d s d A$ (encoding D-serine deaminase) displays a prolonged log phase when grown in urine and a supposed hyper-colonization phenotype in a competitive murine UTI model (Anfora et al., 2007, 2008). Contrary to this, a subsequent study demonstrated that the $d s d A$ deletion does not lead to hyper-colonization and that any gain of fitness phenotype previously reported is likely due to an unrecognized secondary mutation in the sigma factor rpoS gene (Hryckowian et al., 2015). RpoS associates with the core RNA polymerase to control the expression of $\sim 10 \%$ of genes in E. coli, notably when under stressful nutrientlimiting conditions (Weber et al., 2005). This suggests that the unrecognized secondary mutation in rpoS may have affected the $d s d A$ mutant. Although $\mathrm{D}$-serine may play a role as a signaling mechanism for virulence factor expression, and UPEC displays adaptive measures to allow growth in the presence of this bacteriostatic metabolite, evidence suggests that $\mathrm{D}$-serine does not affect the capacity of UPEC to colonize the murine urinary tract (Hryckowian et al., 2015).

\section{Purines and Pyrimidines}

UPEC can also adjust metabolic capacity to maintain growth and colonization in the human bloodstream, subsequently causing urosepsis in a small number of cases. The capacity to defy the bactericidal activity of human serum and colonize the bloodstream represents a crucial virulence advantage for UPEC. It is possible that this virulence advantage could be due to metabolic adaptation: mutagenesis studies identified genes defective in both the de novo purine and pyrimidine nucleotide biosynthetic pathway to be attenuated for commensal E. coli growth in human serum after $24 \mathrm{~h}$ (Samant et al., 2008). This study, making use of the Keio collection of mutants displayed the greatest growth reduction in the purA, purE, pyrC, and pyrE gene deletions when compared to growth in Luria-Bertani 
media (Samant et al., 2008). These defects were complimented and restored when the appropriate nucleotide base precursor was supplemented in the serum. This suggests that nucleotide sugars and precursors are limiting for growth in human serum forcing E. coli to undergo de novo biosynthesis to colonize. Whilst this metabolic adaptation has not been demonstrated for UPEC survival in serum directly, changes in the regulation of purine and pyrimidine synthesis has been observed in the cystitis UTI89 strain (Hadjifrangiskou et al., 2011; discussed below). Thus, the direct role of purine and pyrimidine synthesis for UPEC metabolic adaptation for growth in serum remains an interesting area to explore.

\section{Iron Uptake and Transport Systems}

UPEC express a variety of iron uptake and transport systems such as iron-chelating siderophores and hemophores during intracellular infection. Iron siderophores are low molecular weight metal chelators involved in ferric iron scavenging and play a major role in binding available iron to promote UPEC intracellular colonization within the iron-limiting urine and bladder epithelial cells. Transcriptome studies have identified various genes involved in siderophore synthesis to be dramatically upregulated during in vivo mouse UTI infection, most notably salmochelin and yersiniabactin (Conover et al., 2016). Further, greater production of yersiniabactin and salmochelin in UPEC as opposed to commensal E. coli suggest their possible role in facilitating infection of the urinary tract by UPEC (Henderson et al., 2009). Interestingly, the ferric iron uptake mediator TonB, which interacts with bacterial outer membrane receptors to facilitate iron uptake, has shown to be important in the mouse kidney model of UTI, with a deletion of $\operatorname{ton} B$ resulting in a reduction in UPEC colonization at $48 \mathrm{~h}$ post-infection when compared to wild type (Torres et al., 2001). This highlights the necessity of iron uptake and transport systems in UPEC pathogenesis.

The withholding of free iron by the host is usually mediated by iron-binding proteins like lactoferrin, ferritin, transferrin, ovalbumin and siderocalin (also known as lipocalin-2) (Miethke and Marahiel, 2007). In an effort to counteract bacterial ironscavenging proteins, the host protein siderocalin, introduced into the urinary tract by leukocytes and uroepithelial cells, can restrict the accessibility of iron to UPEC by scavenging ferric iron complexes (Goetz et al., 2002). Siderocalin shows very high affinity to the bacterial siderophore enterobactin, which is found ubiquitously in E. coli strains. However, the lower binding affinity of siderocalin to other siderophores such as aerobactin, salmochelin, and yersinibactin which are more often associated with UPEC strains (Fischbach et al., 2006; Henderson et al., 2009), may provide an evolutionary advantage for UPEC during host iron scavenging. Indeed, purified siderocalin is capable of effectively inhibiting E. coli K-12 that produces only enterobactin. However, no effect was observed on the growth of E. coli that produces all siderophores, such as UPEC (Garénaux et al., 2013).

The composition of human urine plays a role in the regulation of siderocalin activity: an as-yet-unidentified urine constituent inhibits the host siderocalin-mediated growth restriction, allowing the bacterial siderophore enterobactin to overcome the inhibitory iron-binding effects of siderocalin and scavenge host iron (Shields-Cutler et al., 2015). Conversely, the antibacterial activity of siderocalin was found to be associated with urinary $\mathrm{pH}$, with increasing $\mathrm{pH}$ shown to promote siderocalin activity (Shields-Cutler et al., 2015). Other the other side, acidic $\mathrm{pH}$ alone increases the solubility and availability of iron partly contributing to the differences in iron availability in solution and reducing the effect of siderocalin on bacterial siderophores. Metabolomics studies identified the association of increased urinary aryl sulfate metabolites, independent of the urinary $\mathrm{pH}$, with the antibacterial activity of siderocalin (ShieldsCutler et al., 2015). The involvement of urinary constituents in controlling the bacterial growth points to a urinary tract-specific host-pathogen interaction system based on host metabolites and bacterial siderophore biosynthesis during UTIs (Shields-Cutler et al., 2015). This indicates that the host siderocalin uses urinary metabolites as cofactors for restricting the availability of iron to E. coli (Shields-Cutler et al., 2015) and that niche-specific roles of siderophores drive their diversity among UPEC (Chaturvedi et al., 2014; Shields-Cutler et al., 2015). Thus, whilst UPEC has developed adaption strategies to utilize metabolites present in host urine, these metabolites can also be utilized by the host as a defense mechanism against UPEC infection.

\section{Other Known Metabolic Regulators of UPEC Pathogenesis}

As well as the metabolic adaptation to survive and thrive within the above environments, nutritional sensing is vital for UPEC to efficiently respond to nutrient-fluctuating conditions within the host environment, with several regulatory systems, as discussed below, reported to be important for UPEC virulence.

\section{RyhB}

UPEC not only requires to upregulate the genes required for ironacquisition under iron-limited conditions of the urinary tract, but also needs to precisely maintain the levels of iron required for survival. The sRNA RyhB and the global ferric uptake regulator Fur have been implicated for their role in iron acquisition and controlling the storage of iron in E. coli (Massé and Gottesman, 2002). Fur and RyhB regulate the activity of cellular processes such as metabolic pathways, with TCA cycle enzymes known to be targeted by RyhB in E. coli (Massé et al., 2005). RyhB is important for colonization of the urinary tract by UPEC strain CFT073 via the production of iron-acquisition systems, with a $\triangle r y h B$ mutant producing lower levels of the siderophores aerobactin, salmochelin, and enterobactin (Porcheron et al., 2014). Therefore, RyhB is shown to play a role in virulence of the UPEC strain CFT073 (Porcheron et al., 2014).

\section{BarA-UvrY}

The two component system BarA-UvrY responds to nutritional fluctuations through the action of regulatory RNAs CsrB and $\mathrm{CsrC}$ and CsrA protein activity, which regulates expression of genes involved in gluconeogenesis and glycolysis (Romeo, 1998; Suzuki et al., 2002; Weilbacher et al., 2003). Loss of the response regulator UvrY results in decreased fitness in both monkey cystitis and murine ascending UTI competition 
models (Tomenius et al., 2006; Palaniyandi et al., 2012), and decreased invasion of uroepithelial cells (Palaniyandi et al., 2012). Thus, the ability to effectively sense and utilize the mixture of gluconeogenic and glycolytic carbon sources within urine is a key metabolic adaption for UPEC survival.

\section{QseBC}

The two-component regulatory pathway system QseBC, consists of QseC which functions as a sensory kinase to the environment stimuli and host signal, resulting in phosphorylatation of the transcription factor QseB, acting as the response regulator in UPEC. This has been demonstrated by analyzing the qseC mutant, which creates a positive feedback loop constantly expressing QseB (Hadjifrangiskou et al., 2011). Furthermore, deletion of $q s e C$ in the cystitis isolate UTI89 leads to attenuated virulence and altered expression of 36 regulators, 52\% of which modulate at least 18 metabolic pathways (Hadjifrangiskou et al., 2011). These include an increase in pyrimidine production and utilization, whilst purine biosynthetic genes, purK, purM and purT, as well as arginine synthesis genes, $\arg F, \arg G$, and $\arg R$ show downregulation (Hadjifrangiskou et al., 2011). Bypassing purine production by the addition of guanosine or adenine monophosphate was found to restore growth in a qseC mutant, highlighting the importance of purine metabolism for UPEC survival. This is interesting as de novo purine biosynthesis has been recently shown to be essential for proliferation of UPEC within the bladder during IBC, with $\triangle p u r F$ and $\triangle c u p A-p u r F$ operon displaying impaired fitness at 3,6 , and $16 \mathrm{~h}$ post-infection in vivo (Shaffer et al., 2017).

\section{fnr}

Virulence gene expression also needs to be coordinated in response to the availability of oxygen during oxygen-limiting conditions within the bladder (Marteyn et al., 2010). Within UPEC, the transcriptional regulator f $n r$ controls oxygen-related gene expression under anaerobic conditions. This regulator, which contains a DNA-binding domain, was first identified when the fnr mutant was no longer able to use fumarate and nitrate as substrates during anaerobic growth (Lambden and Guest, 1976), and also results in reduced metabolism of $\alpha$-ketoglutarate under oxygen-limited conditions (Barbieri et al., 2014).

Evidence suggests that, in addition to acting as an aerobicanaerobic metabolic regulator for UPEC, fnr controls virulence gene expression within the bladder environment (Lambden and Guest, 1976). The deletion of $f n r$ within UPEC has shown a decreased adherence and subsequently invasion capacity within the epithelial cells of the kidneys and bladder when compared to wild-type (Barbieri et al., 2014), due to multiple virulence factor modifications such as a reduced expression of type 1 pili and $P$ fimbriae (Barbieri et al., 2014). In addition to this, the fnr regulatory homolog within Salmonella has shown evidence of positive virulence expression involved in cell flagella synthesis and motility during pathogenesis (Contreras et al., 1997; Fink et al., 2007). Therefore, fnr contributes to the pathogenesis of UPEC by regulating the expression of important virulence genes (Barbieri et al., 2014) in response to metabolism under different aerobic conditions.

\section{Pst}

The loss of the phosphate-specific transport system (Pst), encoded by the operon pstSCAB-phoU, results in the constitutive activation of the Pho regulon, involved in phosphate transport and metabolism. This constitutive activation has a negative effect on UPEC pathogenicity, with a $\triangle p s t$ CFT073 mutant displaying a reduced fitness in a murine UTI model (Crépin et al., 2012). This effect on virulence is thought to be due to the decreased production of type 1 fimbriae in a $\Delta p s t$ mutant, resulting in decreased invasion of bladder epithelial cells (Crépin et al., 2012), and demonstrates a link between nutrient sensing and UPEC pathogenicity.

\section{LINKING NICHE-SPECIFIC NUTRIENTS TO THE ADAPTATION STRATEGIES OF UPEC}

There is now mounting evidence that the metabolic flexibility of the uropathogen UPEC is crucial for its ability to cause infection of the urinary tract. This is due to the advancements to understand the metabolome of human urine, resulting in the creation of the human urine metabolome database, and numerous studies that have detailed the effect of the loss of a single metabolic enzyme on the fitness of UPEC during urinary tract infection. Whilst this has provided valuable information about the metabolic response of UPEC during infection, understanding the complete metabolic requirements of UPEC in the urinary tract is currently challenging due to each metabolomics and genetic mutational studies being carried out in isolation. Thus, major conclusions correlating the levels of metabolites present in the host environment, and the role of the corresponding bacterial metabolic pathway involved in its catabolism are hard to draw. Ideally, whole-cell "omics" approaches, including transcriptomics and metabolomics, should be carried out in concert with single gene deletion studies to fully correlate the metabolic response with the virulence of UPEC during infection. Using such approaches will also minimize the variations due to sampling procedures, storage conditions and environmental influences. For example, analyzing the concentration of D-serine in urine, and correlating this to the role of $d s d A$ (encoding D-serine deaminase) in fitness during UTI infection will allow us to further understand the direct contribution that amino acid utilization plays during UPEC metabolic adaptation.

New discoveries due to the advancement of "omics" technologies such as metabolomics are also shaping our understanding how UPEC interacts and adapts to nutritionally distinct environments as it moves through the gastrointestinal and urinary tracts. This data is imperative for the identification of new disease biomarkers to improve the diagnosis of UTIs (Mendrick and Schnackenberg, 2009) and for the discovery of novel antibiotic drug targets (Wishart, 2016). However, despite the potential power that metabolomics approaches have in resolving differences pertinent to disease processes ( $\mathrm{Lv}$ et al., 2011), in comparison to other "omics" research (genomics and proteomics), metabolomics still struggles to cover even a small section of the entire metabolome, having identified $<1 \%$ of the 
known human metabolome (Bouatra et al., 2013). Furthermore, due to the complexity of interconnectivity of metabolic processes, analysis of data obtained from metabolomics studies can prove to be difficult to interpret. Regardless, of these shortcomings, to date, metabolomics has successfully allowed comparison between UPEC and commensal E. coli, providing novel targets for virulence-based diagnosis (Su et al., 2016a). These virulence factors can be targeted to control the UPEC pathogenic mechanisms, thereby serving as potential drug targets (Hannan et al., 2012; Kostakioti et al., 2012; Subashchandrabose et al., 2014; Flores-Mireles et al., 2015; Yan et al., 2015; Su et al., 2016a,b).

\section{METABOLISM AS A POTENTIAL ANTIBACTERIAL TARGET FOR UTIS \\ Drug Targets}

Most of the presently used antimicrobial drugs target a limited number of bacterial processes, which are essential under nutrient-rich conditions. However, growth in a nutrient-rich medium in a laboratory setting does not reflect conditions within the host, wherein UPEC must co-exist with gut microbes for available nutrients, as well as display metabolic flexibility to adapt to the harsh and comparatively nutrient-scarce urinary tract environment. Under such circumstances, UPEC has developed novel strategies through metabolic adaptations in order to adjust and survive the challenging environment of the urinary tract.
New drugs to be developed must target adaptive strategies of UPEC that are essential for survival in the host environment; for example, the metabolic pathways shown to be important for UPEC pathogenesis (Figure 2). Indeed, the longest standing antibiotic class of sulfonamides target the metabolic process of folate synthesis (Bourne, 2014), showing precedent for designing drugs to target bacterial metabolism. Investigation of metabolic pathways necessary for growth in a nutrient-restricted medium serve as a better proxy for infection-causing conditions inside the host, and has identified the essentiality of synthesis of amino acids, nucleotides and vitamins (Côté et al., 2016); therefore such processes are potential drug targets (Figure 3).

Under the nutrient stress conditions of the urinary tract, genetic and metabolic adaptation studies of UPEC has shown that central metabolic pathways are necessary for fitness of UPEC in vivo, and amino acids and peptides serve as the main source of carbon for UPEC during the urinary tract infection (Alteri et al., 2009). These pathways therefore form a critical component of the virulence of pathogenic microorganisms (Alteri et al., 2009, 2015; Lv et al., 2011, 2014; Yan et al., 2015), and serve as potential drug targets. For example, tpiA, which encodes triosephosphate isomerase involved in glycolysis, is important for kidney infection by UPEC (Alteri et al., 2009) and could therefore be a potential drug target for the treatment of pyelonephritis.

Urine, being nutritionally restrictive, is unable to provide all the growth factors required for bacterial growth, several of which are synthesized de novo by bacteria that are adapted to

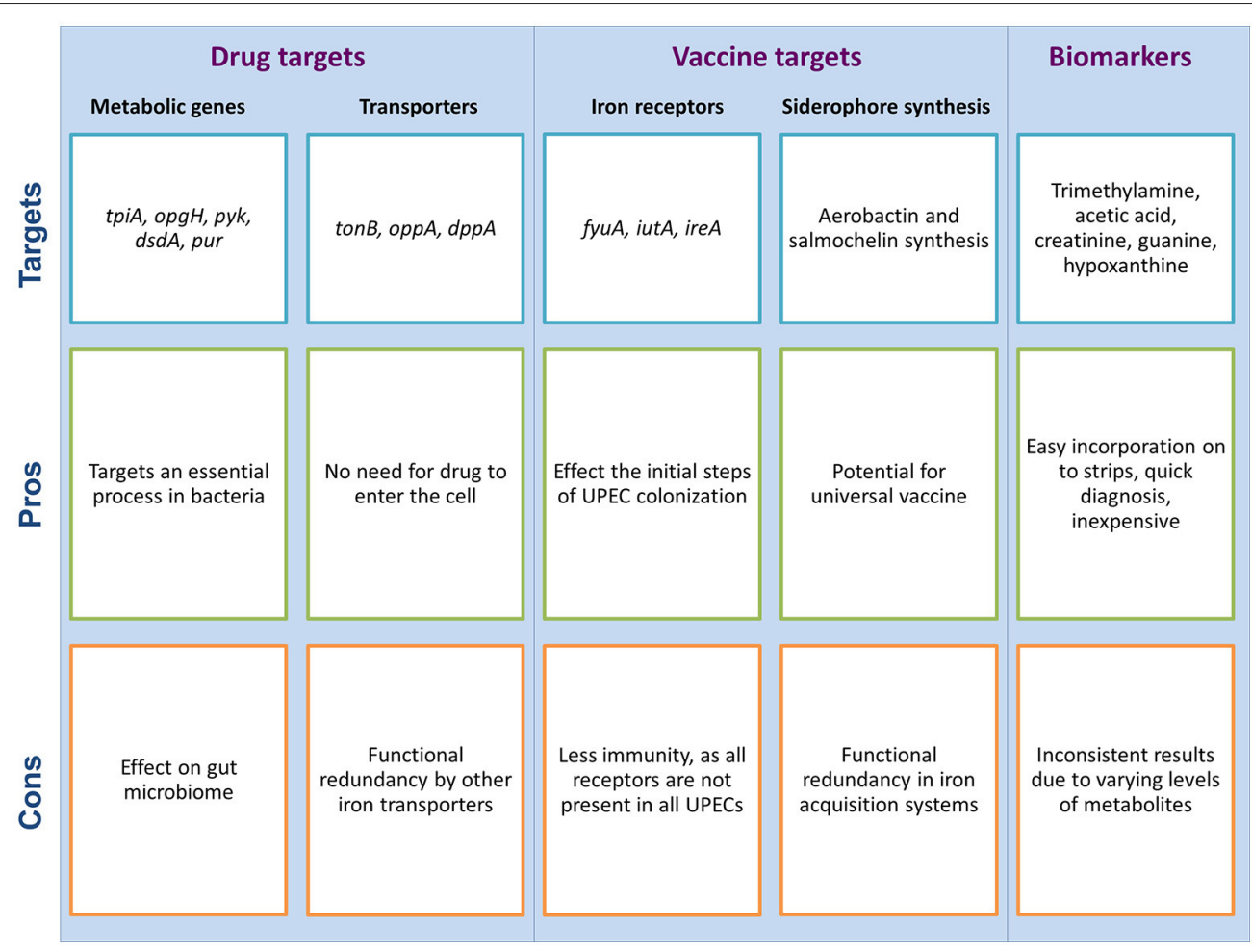

FIGURE 3 | Metabolism as a potential target for the development of new drugs and vaccines for UTIs, and the use of urine as a diagnostic fluid for UTI detection; each with potential pros and cons associated with them. These potential targets are suggested by the authors as per the literature search. 
survival in this environment. Inhibitors of the synthesis process of these growth factors may therefore have therapeutic value for prophylaxis in the case of recurrent UTIs or for people with high risk factors for infection (e.g., during pregnancy), as well as treatment of uncomplicated UTIs (Hull and Hull, 1997). For instance, biosynthetic pathways involved in the synthesis of carbohydrates, purines and pyrimidines, and energy metabolism, those involved in D-serine utilization ( $d s d$ locus) and uptake of iron $(\operatorname{ton} B)$, are important factors contributing to bacterial fitness in vivo. Antibiotics that target these metabolic pathways or uptake systems hold the potential for treatment of UTIs (Vejborg et al., 2012).

\section{Vaccines}

UTIs have a high recurrence rate of 25\% (Bauckman and Mysorekar, 2016) and can occur in an individual for the duration of their lifetime, which requires long-term prophylaxis with antimicrobials (Kodner and Thomas Gupton, 2010; Kostakioti et al., 2012). Therefore, prevention using vaccines against uropathogens would be a promising alternative, especially for high-risk populations such as immunocompromised people and pregnant women (Brumbaugh et al., 2013).

Iron-acquisition strategies of UPECs are of interest for the development of novel diagnostics and therapeutics for UTIs (Miethke and Marahiel, 2007). The continuous struggle for iron between the host and the pathogen has spurred the ironrelated coevolution of host and the pathogen as a defense and antidefense strategy by the two. This coevolution had resulted in the development of siderophore-neutralization strategies by the host in order to suppress the pathogen multiplication and reutilization strategies by the pathogen. Additionally, hosts have also developed cell conversion pathways and siderophorebased iron delivery systems to break the pathogen's supply of iron. Successful treatment strategies could therefore focus on weakening the iron-acquisition systems of UPECs or improving the siderophore-neutralization strategies of the host.

This is an area of interest for vaccine development not just restricted to UTIs, and has led to the development of vaccines targeting some iron acquisition proteins; like yersiniabactin uptake receptor FyuA, iron uptake aerobactin receptor IutA and iron-responsive siderophore receptor IreA (Brumbaugh et al., 2013), and siderophores yersiniabactin and aerobactin (Mike et al., 2016). However, targeting a single iron receptor is unlikely to yield a broad level of immunity because iron acquisition mechanisms show functional redundancy in E. coli (Garcia et al., 2011), and not all receptors are found in all UPEC strains (Brumbaugh et al., 2013). Therefore, an iron receptorbased multi-epitope vaccine against UPEC has the potential to provide broad-spectrum immunization, as has been developed for ExPEC, which has shown two-fold reduction in the bacterial load in a mouse peritonitis model (Wieser et al., 2010). It is possible that a similar multi-epitope vaccine would provide protection against diverse UPEC strains.

Siderophore synthesis is identified to modulate the differential metabolome of UPEC and non-UPEC strains, therefore the genes involved in synthesis of these siderophores are candidate targets for the development of drugs and vaccines against
UPECs (Su et al., 2016a). Siderophores, which are traditionally considered an important virulence factor for UTI (Chaturvedi et al., 2014), have been recently demonstrated to enhance the virulence of UPEC partially by modulating their amino acid metabolism (Su et al., 2016a). Recent evidence has shown that the siderophores aerobactin and salmochelin present in UPEC have a stronger modulatory effect than other siderophores on amino acid metabolism (Su et al., 2016b). Therefore, siderophore biosynthesis and its associated metabolic pathways may be novel targets for therapeutics and virulence-based diagnoses.

\section{Diagnosis}

The metabolic profile of infected urine, as analyzed by metabolomics, may reveal promising biomarkers to be used for the diagnosis of UTIs. Several examples have already been identified, including trimethylamine and acetic acid, which have potential for incorporation on to a dip strip (Karlsen and Dong, 2015). Another example is creatinine, a breakdown product of creatine, defined as a biomarker for the diagnosis of nephropathy (Ariza-Heredia et al., 2014). Higher creatine concentrations are observed in UPEC-grown urine in comparison to non-UPEC strain, therefore it may be used as a biomarker for the diagnosis of UPEC infection (Su et al., 2016a). Similarly, metabolites related to purine and pyrimidine metabolism are upregulated in UPECs as compared to non-UPECs, for example guanine and hypoxanthine (Su et al., 2016a). These two metabolites are known to be critical for the virulence of uropathogens (Vejborg et al., 2012; Karlsen and Dong, 2015), therefore hypoxanthine has the potential to be used as a biomarker for the diagnosis of UTIs. However, these potential markers for determining UTIs are from studies performed by culturing UPEC in urine obtained from healthy individuals under in vitro conditions (Karlsen and Dong, 2015), or by comparing the profile of metabolites produced by UPECs and non-UPECs under in vitro conditions ( $\mathrm{Su}$ et al., 2016a), which are settings that are in the absence of host immune and cellular responses. The urinary composition of a healthy individual is highly likely to be very metabolically different from that of a UTI patient, partly due to the inflammatory response of the host. Furthermore, much of the work to establish the role of metabolism during UPEC infection has been performed using various mouse models, which raises concerns about the conservation of metabolic responses as compared to human infection. These factors together raises questions on using these identified metabolites as a means of determining UTIs. Thus, future studies should focus on understanding the metabolomic profile of urine from UPEC-infected individuals to provide reliable data for the identification of biomarkers of UTIs in response to the host immune response.

\section{Pros and Cons of Using Metabolism as an Antibacterial Target}

A number of studies have explored the urine metabolome of a healthy person and there are many examples comparing metabolite levels in a healthy person to a diseased person, thereby drawing conclusions about the role of specific metabolites in progression of a disease (Alonso et al., 2016; Feng et al., 2016). To our knowledge, none of the suggested metabolites from those 
studies have been standardized for use as a diagnostic marker for UTIs or developed as a potential drug target. This is most likely due to the complexity of metabolism itself, which makes it hard to get reliable and reproducible data required for any metabolite to be used as a disease marker, or any metabolic enzyme to be used as a drug target. Urine metabolomics analysis is usually done on pooled urine samples, as done in the Human urine metabolome project (Bouatra et al., 2013). This provides information about the metabolome of the combined urine sample obtained from different individuals rather than the urinary composition of one individual. Therefore, the levels of metabolites obtained from such studies do not hold a true potential to be standardized for the determination of UTIs. Further complicating is the fact that the levels of metabolites can vary merely due to different physiological conditions of a person which is unrelated to disease status (discussed in the next paragraph). Therefore, the parameters for threshold levels of metabolites must be carefully considered before determining their potential for UTI diagnosis.

Although there are a number of potential biomarkers identified through individual studies, one very critical point to consider before selecting such metabolites as a suitable UTI biomarker is that the concentration of certain metabolite can naturally vary by as much as seven-fold in different individuals, depending on a number of factors like age, gender, health status, diet and activity level (Saude et al., 2007; Bouatra et al., 2013). Overall, dietary intake appears to be the most influential factor on the concentrations of urinary metabolites, with the type of diet dictating the metabolite concentration. For example, mannitol, which is commonly present in foods like carrots, apples, pineapples and asparagus, is commonly found in human urine because of its poor absorption by the body (Bouatra et al., 2013). Similarly, chocolate and meat intake are known to increase the concentrations of polyphenolderived phenolic acids and 1-methylhistidine, respectively, in the urine (Sjölin et al., 1987; Rios et al., 2003). Some genderrelated effects are shown to influence the level of creatinine, a major component of the human urine and often used to normalize metabolite concentrations of urine samples. Increased intake of creatine or a protein-rich diet is also shown to increase the urinary excretion of creatinine (Taylor et al., 1989). In addition to dietary influence on the urine metabolome, the levels of metabolites in urine vary with the physiological status; for example, the levels of 3-hydroxybutyric acid increases during fasting (Bouatra et al., 2013). Therefore, a careful consideration of patient gender, diet and physiological status is necessary before drawing conclusions about any potential UTI diagnostic biomarker. Moreover, further studies need to be conducted to investigate the contribution of each of these factors to the urine metabolome. However, such studies would require a significant amount of technical expertise, considering the diversity of urinary metabolites and the range of factors contributing toward the variation in levels of those metabolites. In addition to exploring the contribution of such factors to the urine metabolome, conditions will need to be standardized for the collection of samples for examination. For example, baseline concentrations of the biomarker may vary if the urine sample is collected under fasting conditions as compared to non-fasting conditions, which has implications in the diagnosis of a UTI.

One important point to consider when developing drugs that target the process of metabolism, or for that matter, for all drugs that target essential bacterial processes, is the potential side effects on the balance of the microbiome in the gut. Therefore, ideally, the target to be selected for drug development should be specific to UPEC and should have minimal effect on the gut microbiota. Since the availability of iron to UPEC is limiting in the urinary tract, the uptake of iron using ton $B$ transporter is important for UPEC virulence. Therefore, the iron transporters in UPEC are potential drug targets. However, for this target to be practically viable, further research needs to be done toward investigating the role of iron transporters for intestinal survival of the members of gut microbiome, as the potential drug will have consequences on the gut microbiome as well.

Despite potential shortcomings with using metabolomics as an antibacterial target, the essentiality of metabolism for bacterial survival and the requirement of its adaptation inside the urinary tract make it a promising target for UPEC treatment. However, the current knowledge of the urine metabolome is incomplete, but further advancements in techniques to fully quantify metabolites and their levels in urine will aid our understanding of the human urine metabolome. In concert, studies are being continuously done to explore the role of metabolic enzymes in UPEC adaptation inside the urinary tract and new metabolic enzymes are being discovered. Identification of these metabolic enzymes and the roles they play in UPEC adaptation, along with correlating those adaptation strategies to the levels of corresponding metabolites in both the bacterial cell and human urine will allow us to better identify novel drug targets aimed specifically at UPEC metabolism.

\section{CONCLUSION}

UTIs are one of the most prevalent bacterial infections, resulting in billions of dollars of expenditure every year (Stamm and Norrby, 2001). Of greater concern however is the increasing issue of antibiotic resistance amongst pathogens causing UTIs (FloresMireles et al., 2015). Metabolism is an essential bacterial process and has been shown to be critical for UPEC adaptation to specific host environments during infection, making it an ideal potential treatment target. Further, identifying the metabolic adaptation strategies of UPEC strains can help uncover an entirely new area with prospective exploitation for the development of novel antimicrobials to treat UTIs.

Our current understanding of the urine metabolome and UPEC metabolic adaptation is incomplete, partially due to technological shortcomings and the complexity and quantity of metabolites produced by UPEC, especially in response to interaction with the host environment. However, there have been recent efforts to understand the contribution of metabolism to UPEC virulence. From the current knowledge, limited availability of iron in urine appears to be the growth-limiting factor for UPEC growth in human urine; therefore UPEC systems involved in the uptake and utilization of iron seem to be a very promising target for novel drug development. The most abundant amino acid known to be present in urine is $\mathrm{D}$-serine, which has been 
shown to be important for the virulence of UPEC (Vejborg et al., 2012). Therefore, the systems involved in catabolism of this amino acid also can be potential drug targets. Further advancements in "omics" techniques, along with a multi-omics approach, will provide better understanding of the adaptation strategies of UPECs and will guide us in future treatment developments.

\section{AUTHOR CONTRIBUTIONS}

$\mathrm{RM}$ and $\mathrm{AB}$ conceived the manuscript. $\mathrm{RM}, \mathrm{DM}, \mathrm{ID}$, and $\mathrm{AB}$ wrote the manuscript. ID, $\mathrm{EH}$, and $\mathrm{AB}$ edited the manuscript.

\section{REFERENCES}

Alonso, A., Julià, A., Vinaixa, M., Domènech, E., Fernández-Nebro, A., Cañete, J. D., et al. (2016). Urine metabolome profiling of immune-mediated inflammatory diseases. BMC Med. 14:133. doi: 10.1186/s12916-016-0681-8.

Alteri, C. J., Himpsl, S. D., and Mobley, H. L. T. (2015). Preferential use of central metabolism in vivo reveals a nutritional basis for polymicrobial infection. PLoS Pathog. 11:e1004601. doi: 10.1371/journal.ppat.1004601

Alteri, C. J., and Mobley, H. L. T. (2012). Escherichia coli physiology and metabolism dictates adaptation to diverse host microenvironments. Curr. Opin. Microbiol. 15, 3-9. doi: 10.1016/j.mib.2011.12.004

Alteri, C. J., Smith, S. N., and Mobley, H. L. T. (2009). Fitness of Escherichia coli during urinary tract infection requires gluconeogenesis and the TCA cycle. PLoS Pathog. 5:e1000448. doi: 10.1371/journal.ppat.1000448

Andersen, T. E., Khandige, S., Madelung, M., Brewer, J., Kolmos, H. J., and MøllerJensen, J. (2012). Escherichia coli uropathogenesis in vitro invasion, cellular escape, and secondary infection analyzed in a human bladder cell infection model. Infect. Immun. 80, 1858-1867. doi: 10.1128/IAI.06075-11

Anderson, G. G., Palermo, J. J., Schilling, J. D., Roth, R., Heuser, J., and Hultgren, S. J. (2003). Intracellular bacterial biofilm-like pods in urinary tractiInfections. Science 301, 105-107. doi: 10.1126/science. 1084550

Anfora, A. T., Halladin, D. K., Haugen, B. J., and Welch, R. A. (2008). Uropathogenic Escherichia coli CFT073 is adapted to acetatogenic growth but does not require acetate during murine urinary tract infection. Infect. Immun. 76, 5760-5767. doi: 10.1128/IAI.00618-08

Anfora, A. T., Haugen, B. J., Roesch, P., Redford, P., and Welch, R. A. (2007). Roles of serine accumulation and catabolism in the colonization of the murine urinary tract by Escherichia coli CFT073. Infect. Immun. 75, 5298-5304. doi: 10.1128/IAI.00652-07

Ariza-Heredia, E. J., Beam, E. N., Lesnick, T. G., Cosio, F. G., Kremers, W. K., and Razonable, R. R. (2014). Impact of urinary tract infection on allograft function after kidney transplantation. Clin. Transplant. 28, 683-690. doi: $10.1111 / \mathrm{ctr} .12366$

Asadi, S., Kargar, M., Solhjoo, K., Najafi, A., and Ghorbani-Dalini, S. (2014). The association of virulence determinants of uropathogenic Escherichia coli with antibiotic resistance. Jundishapur J. Microbiol. 7:e9936. doi: 10.5812/jjm.9936

Barbieri, N. L., Nicholson, B., Hussein, A., Cai, W., Wannemuehler, Y. M., Dell'Anna, G., et al. (2014). FNR regulates expression of important virulence factors contributing to pathogenicity of uropathogenic Escherichia coli. Infect. Immun. 82, 5086-5098. doi: 10.1128/IAI.02315-14

Bauckman, K. A., and Mysorekar, I. U. (2016). Ferritinophagy drives uropathogenic Escherichia coli persistence in bladder epithelial cells. Autophagy 12, 850-863. doi: 10.1080/15548627.2016.1160176

Bergstrom, K. S., Sham, H. P., Zarepour, M., and Vallance, B. A. (2012). Innate host responses to enteric bacterial pathogens: a balancing act between resistance and tolerance. Cell Microbiol. 14, 475-484. doi: 10.1111/j.1462-5822.2012.01750.x

Bouatra, S., Aziat, F., Mandal, R., Guo, A. C., Wilson, M. R., Knox, C., et al. (2013). The human urine metabolome. PLOS ONE 8:e73076. doi: 10.1371/journal.pone.0073076
All authors provided comments during the preparation of the manuscript.

\section{ACKNOWLEDGMENTS}

The authors are grateful to Mark Schembri for helpful discussions. RM and DM are supported by an Australian Government Research Training Program Scholarship. ID is supported by the Australian Research council (DP160101076/FT160100010). AB and EH are supported by an Australian Research Council Discovery project grant DP150102062.

Bourne, C. R. (2014). Utility of the biosynthetic folate pathway for targets in antimicrobial Discovery. Antibiotics 3, 1-28. doi: 10.3390/antibiotics3010001

Brown, S. A., Palmer, K. L., and Whiteley, M. (2008). Revisiting the host as a growth medium. Nat. Rev. Microbiol. 6, 657-666. doi: 10.1038/nrmicro1955

Brückner, H., Haasmann, S., and Friedrich, A. (1994). Quantification of D-amino acids in human urine using GC-MS and HPLC. Amino Acids 6, 205-211. doi: $10.1007 / \mathrm{BF} 00805848$

Brumbaugh, A. R., Smith, S. N., and Mobley, H. L. T. (2013). Immunization with the yersiniabactin receptor, FyuA, protects against pyelonephritis in a murine model of urinary tract infection. Infect. Immun. 81, 3309-3316. doi: 10.1128/IAI.00470-13

Chang, D.-E., Smalley, D. J., Tucker, D. L., Leatham, M. P., Norris, W. E., Stevenson, S. J., et al. (2004). Carbon nutrition of Escherichia coli in the mouse intestine. Proc. Natl. Acad. Sci. U.S.A. 101, 7427-7432. doi: 10.1073/pnas.0307888101

Chaturvedi, K. S., Hung, C. S., Giblin, D. E., Urushidani, S., Austin, A. M., Dinauer, M. C., et al. (2014). Cupric yersiniabactin is a virulence-associated superoxide dismutase mimic. ACS Chem. Biol. 9, 551-561. doi: 10.1021/cb400658k

Conover, M. S., Hadjifrangiskou, M., Palermo, J. J., Hibbing, M. E., Dodson, K. W., and Hultgren, S. J. (2016). Metabolic requirements of Escherichia coli in intracellular bacterial communities during urinary tract infection pathogenesis. MBio 7, e00104-e00116. doi: 10.1128/mBio.00104-16

Contreras, I., Toro, C. S., Troncoso, G., and Mora, G. C. (1997). Salmonella typhi mutants defective in anaerobic respiration are impaired in their ability to replicate within epithelial cells. Microbiology 143, 2665-2672. doi: 10.1099/00221287-143-8-2665

Conway, T., and Cohen, P. S. (2015). Commensal and pathogenic Escherichia coli metabolism in the gut. Microbiol. Spectr. 3:MBP-0006-2014. doi: 10.1128/ microbiolspec.MBP-0006-2014

Conway, T., Krogfelt, K. A., and Cohen, P. S. (2004). "Chapter 8.3.1.2, The life of commensal Escherichia coli in the mammalian intestine," in EcoSal Plus Cellular and molecular biology of E. coli Salmonella, and the Enterobacteriaceae, 3rd Edn., ed J. B. Kaper (Washington, DC: ASM Press).

Cosloy, S. D., and McFall, E. (1973). Metabolism of D-serine in Escherichia coli K-12: mechanism of growth inhibition. J. Bacteriol. 114, 685-694.

Côté, J.-P., French, S., Gehrke, S. S., MacNair, C. R., Mangat, C. S., Bharat, A., et al. (2016). The genome-wide interaction network of nutrient stress genes in Escherichia coli. MBio 7, 1-11. doi: 10.1128/mbio.01714-16

Crépin, S., Houle, S., Charbonneau, M. E., Mourez, M., Harel, J., and Dozois, C. M. (2012). Decreased expression of type 1 fimbriae by a pst mutant of uropathogenic Escherichia coli reduces urinary tract infection. Infect. Immun. 80, 2802-2815. doi: 10.1128/IAI.00162-12

Eisenreich, W., Dandekar, T., Heesemann, J., and Goebel, W. (2010). Carbon metabolism of intracellular bacterial pathogens and possible links to virulence. Nat. Rev. Microbiol. 8, 401-412. doi: 10.1038/nrmicro2351

Feng, Q., Liu, Z., Zhong, S., Li, R., Xia, H., Jie, Z., et al. (2016). Integrated metabolomics and metagenomics analysis of plasma and urine identified microbial metabolites associated with coronary heart disease. Sci. Rep. 6:22525. doi: 10.1038/srep22525 
Fink, R. C., Evans, M. R., Porwollik, S., Vazquez-Torres, A., Jones-Carson, J., Troxell, B., et al. (2007). FNR is a global regulator of virulence and anaerobic metabolism in Salmonella enterica serovar typhimurium (ATCC 14028s). J. Bacteriol. 189, 2262-2273. doi: 10.1128/JB.00726-06

Fischbach, M. A., Lin, H., Zhou, L., Yu, Y., Abergel, R. J., Liu, D. R., et al. (2006). The pathogen-associated iroA gene cluster mediates bacterial evasion of lipocalin 2. Proc. Natl. Acad. Sci. U.S.A. 103, 16502-16507. doi: 10.1073/pnas.0604636103

Flores-Mireles, A. L., Walker, J. N., Caparon, M., and Hultgren, S. J. (2015). Urinary tract infections: epidemiology, mechanisms of infection and treatment options. Nat. Rev. Microbiol. 13, 269-284. doi: 10.1038/nrmicro3432

Foxman, B. (2002). Epidemiology of urinary tract infections: incidence, morbidity, and economic costs. Am. J. Med. 113, 5-13. doi: 10.1016/S0002-9343(02)01054-9

Foxman, B. (2003). Epidemiology of urinary tract infections: incidence, morbidity, and economic costs. Disease-a-Month 49, 53-70. doi: 10.1067/mda.2003.7

Freter, R., Brickner, H., Fekete, J., Vickerman, M. M., and Carey, K. E. (1983). Survival and implantation of Escherichia coli in the intestinal tract. Infect. Immun. 39, 686-703.

Garcia, E. C., Brumbaugh, A. R., and Mobley, H. L. T. (2011). Redundancy and specificity of Escherichia coli iron acquisition systems during urinary tract infection. Infect. Immun. 79, 1225-1235. doi: 10.1128/IAI.01222-10

Garénaux, A., Houle, S., Folch, B., Dallaire, G., Truesdell, M., Lépine, F., et al. (2013). Avian lipocalin expression in chickens following Escherichia coli infection and inhibition of avian pathogenic Escherichia coli growth by Ex-FABP. Vet. Immunol. Immunopathol. 152, 156-167. doi: 10.1016/j.vetimm.2012.09.018

Gielda, L. M., and DiRita, V. J. (2012). Zinc competition among the intestinal microbiota. MBio 3, e00171-e00112. doi: 10.1128/mBio.00171-12

Goetz, D. H., Holmes, M. A., Borregaard, N., Bluhm, M. E., Raymond, K. N., and Strong, R. K. (2002). The neutrophil lipocalin NGAL is a bacteriostatic agent that interferes with siderophore-mediated iron acquisition. Mol. Cell 10, 1033-1043. doi: 10.1016/S1097-2765(02)00708-6

Hadjifrangiskou, M., Kostakioti, M., Chen, S. L., Henderson, J. P., Greene, S. E., and Hultgren, S. J. (2011). A central metabolic circuit controlled by QseC in pathogenic Escherichia coli. Mol. Microbiol. 80, 1516-1529. doi: 10.1111/j.1365-2958.2011.07660.x

Hannan, T. J., Totsika, M., Mansfield, K. J., Moore, K. H., Schembri, M. A., and Hultgren, S. J. (2012). Host-pathogen checkpoints and population bottlenecks in persistent and intracellular uropathogenic Escherichia coli bladder infection. FEMS Microbiol. Rev. 36, 616-648. doi: 10.1111/j.1574-6976.2012. 00339.x

Henderson, J. P., Crowley, J. R., Pinkner, J. S., Walker, J. N., Tsukayama, P., Stamm, W. E., et al. (2009). Quantitative metabolomics reveals an epigenetic blueprint for iron acquisition in uropathogenic Escherichia coli. PLoS Pathog. 5:e1000305. doi: 10.1371/journal.ppat.1000305

Hooton, T. M. (2012). Uncomplicated urinary tract infection. N. Engl. J. Med. 366, 1028-1037. doi: 10.1056/NEJMcp1104429

Hryckowian, A. J., Baisa, G. A., Schwartz, K. J., and Welch, R. A. (2015). DsdA does not affect colonization of the murine urinary tract by Escherichia coli CFT073. PLoS ONE 10:e0138121. doi: 10.1371/journal.pone.0138121

Hull, R. A., and Hull, S. I. (1997). Nutritional requirements for growth of uropathogenic Escherichia coli in human urine. Infect. Immun. 65, 1960-1961.

Jones, C. H., Pinkner, J. S., Roth, R., Heuser, J., Nicholes, A. V., Abraham, S. N., et al. (1995). FimH adhesin of type 1 pili is assembled into a fibrillar tip structure in the Enterobacteriaceae. Proc. Natl. Acad. Sci. U.S.A. 92, 2081-2085. doi: 10.1073/pnas.92.6.2081

Justice, S. S., Hung, C., Theriot, J. A., Fletcher, D. A., Anderson, G. G., Footer, M. J., et al. (2004). Differentiation and developmental pathways of uropathogenic Escherichia coli in urinary tract pathogenesis. Proc. Natl. Acad. Sci. U.S.A. 101, 1333-1338. doi: 10.1073/pnas.0308125100

Justice, S. S., Hunstad, D. A., Seed, P. C., and Hultgren, S. J. (2006a). Filamentation by Escherichia coli subverts innate defenses during urinary tract infection. Proc. Natl. Acad. Sci. U.S.A. 103, 19884-19889. doi: 10.1073/pnas.06063 29104

Justice, S. S., Lauer, S. R., Hultgren, S. J., and Hunstad, D. A. (2006b). Maturation of intracellular Escherichia coli communities requires SurA. Infect. Immun. 74, 4793-4800. doi: 10.1128/IAI.00355-06
Karlsen, H., and Dong, T. (2015). Biomarkers of urinary tract infections: state of the art, and promising applications for rapid strip-based chemical sensors. Anal. Methods 7, 7961-7975. doi: 10.1039/C5AY01678A

Katouli, M. (2010). Population structure of gut Escherichia coli and its role in development of extra-intestinal infections. Iran. J. Microbiol. 2, $59-72$.

Kodner, C. M., and Thomas Gupton, E. K. (2010). Recurrent urinary tract infections in women: Diagnosis and management. Am. Fam. Phys. 82, 638-643.

Kortman, G. A., Raffatellu, M., Swinkels, D. W., and Tjalsma, H. (2014). Nutritional iron turned inside out: intestinal stress from a gut microbial perspective. FEMS Microbiol. Rev. 38, 1202-1234. doi: 10.1111/1574-6976.12086

Kostakioti, M., Hultgren, S. J., and Hadjifrangiskou, M. (2012). Molecular blueprint of uropathogenic Escherichia coli virulence provides clues toward the development of anti-virulence therapeutics. Virulence 3, 592-594. doi: $10.4161 /$ viru. 22364

Kucheria, R., Dasgupta, P., Sacks, S. H., Khan, M. S., and Sheerin, N. S. (2005). Urinary tract infections: new insights into a common problem. Postgrad. Med. J. 81, 83-86. doi: 10.1136/pgmj.2004.023036

Lambden, P. R., and Guest, J. R. (1976). Mutants of Escherichia coli K12 unable to use fumarate as an anaerobic electron acceptor. J. Gen. Microbiol. 97, 145-160. doi: 10.1099/00221287-97-2-145

Langermann, S., Möllby, R., Burlein, J. E., Palaszynski, S. R., Auguste, C. G., DeFusco, A., et al. (2000). Vaccination with FimH adhesin protects cynomolgus monkeys from colonization and infection by uropathogenic Escherichia coli. J. Infect. Dis. 181, 774-778. doi: 10.1086/315258

Lichtenberger, P., and Hooton, T. M. (2008). Complicated urinary tract infections. Curr. Infect. Dis. Rep. 10, 499-504. doi: 10.1007/s11908-008-0081-0

Lv, H., Hung, C. S., Chaturvedi, K. S., Hooton, T. M., and Henderson, J. P. (2011). Development of an integrated metabolomic profiling approach for infectious diseases research. Analyst 136, 4752-4763. doi: 10.1039/c1an15590c

Lv, H., Hung, C. S., and Henderson, J. P. (2014). Metabolomic analysis of siderophore cheater mutants reveals metabolic costs of expression in uropathogenic Escherichia coli. J. Proteome Res. 13, 1397-1404. doi: $10.1021 / \mathrm{pr} 4009749$

Maltby, R., Leatham-Jensen, M. P., Gibson, T., Cohen, P. S., and Conway, T. (2013). Nutritional basis for colonization resistance by human commensal Escherichia coli strains HS and Nissle 1917 against E. coli O157:H7 in the mouse intestine. PLoS ONE 8:e53957. doi: 10.1371/journal.pone.0053957

Mandal, R., Guo, A. C., Chaudhary, K. K., Liu, P., Yallou, F. S., Dong, E., et al. (2012). Multi-platform characterization of the human cerebrospinal fluid metabolome: a comprehensive and quantitative update. Genome Med. 4, 38-49. doi: $10.1186 / \mathrm{gm} 337$

Marteyn, B., West, N. P., Browning, D. F., Cole, J. A, Shaw, J. G., Palm, F., et al. (2010). Modulation of Shigella virulence in response to available oxygen in vivo. Nature 465, 355-358. doi: 10.1038/nature08970

Martinez, J. J., and Hultgren, S. J. (2002). Requirement of Rho-family GTPases in the invasion of type 1-piliated uropathogenic Escherichia coli. Cell Microbiol. 4, 19-28. doi: 10.1046/j.1462-5822.2002.00166.x

Martinez, J. J., Mulvey, M. A., Schilling, J. D., Pinkner, J. S., and Hultgren, S. J. (2000). Type 1 pilus-mediated bacterial invasion of bladder epithelial cells. EMBO J. 19, 2803-2812. doi: 10.1093/emboj/19.12.2803

Massé, E., and Gottesman, S. (2002). A small RNA regulates the expression of genes involved in iron metabolism in Escherichia coli. Proc. Natl. Acad. Sci. U.S.A. 99, 4620-4625. doi: 10.1073/pnas.032066599

Massé, E., Vanderpool, C. K., and Gottesman, S. (2005). Effect of RyhB small RNA on global iron use in Escherichia coli. J. Bacteriol. 187, 6962-6971. doi: 10.1128/JB.187.20.6962-6971.2005

Mendrick, D. L., and Schnackenberg, L. (2009). Genomic and metabolomic advances in the identification of disease and adverse event biomarkers. Biomark Med. 3, 605-615. doi: 10.2217/bmm.09.43

Miethke, M., and Marahiel, M. A. (2007). Siderophore-based iron acquisition and pathogen control. Microbiol. Mol. Biol. Rev. 71, 413-451. doi: 10.1128/MMBR.00012-07

Mike, L. A., Smith, S. N., Sumner, C. A., Eaton, K. A., and Mobley, H. L. T. (2016) Siderophore vaccine conjugates protect against uropathogenic Escherichia coli urinary tract infection. Proc. Natl. Acad. Sci. U.S.A. 113:201606324. doi: $10.1073 /$ pnas. 1606324113 
Miranda, R. L., Conway, T., Leatham, M. P., Chang, D. E., Norris, W. E., Allen, J. H., et al. (2004). Glycolytic and gluconeogenic growth of Escherichia coli O157: H7 ( EDL933) and E. coli K-12 ( MG1655) in the mouse intestine. Infect. Immun. 72, 1666-1676. doi: 10.1128/IAI.72.3.1666-16 76.2004

Mobley, H. L. T. (2016). Measuring Escherichia coli gene expression during human urinary tract infections. Pathogens 5, 1-9. doi: 10.3390/pathogens5010001

Møller, A. K., Leatham, M. P., Conway, T., Nuijten, P. J. M., De Haan, L. A. M., Krogfelt, K. A., et al. (2003). An Escherichia coli MG1655 lipopolysaccharide deep-rough core mutant grows and survives in mouse cecal mucus but fails to colonize the mouse large intestine. Infect. Immun. 71, 2142-2152. doi: 10.1128/IAI.71.4.2142-21 52.2003

Mulvey, M. A. (2002). Adhesion and entry of uropathogenic Escherichia coli. Cell Microbiol. 4, 257-271. doi: 10.1046/j.1462-5822.2002. 00193.x

Mulvey, M. A., Lopez-Boado, Y. S., Wilson, C. L., Roth, R., Parks, W. C., Heuser, J., et al. (1998). Induction and evasion of host defenses by Type 1-piliated Uropathogenic Escherichia coli. Sci. 282, 1494-1497. doi: $10.1126 /$ science.282.5393.1494

Palaniyandi, S., Mitra, A., Herren, C. D., Lockatell, C. V., Johnson, D. E., Zhu, X., et al. (2012). BarA-UvrY two-component system regulates virulence of uropathogenic E. coli CFT073. PLoS ONE 7:e31348. doi: 10.1371/journal.pone.0031348

Patti, G. J., Yanes, O., and Siuzdak, G. (2012). Innovation: metabolomics: the apogee of the omics trilogy. Nat. Rev. Mol. Cell Biol. 13, 263-269. doi: $10.1038 / \mathrm{nrm} 3314$

Pätzold, R., Schieber, A., and Brückner, H. (2005). Gas chromatographic quantification of free D-amino acids in higher vertebrates. Biomed. Chromatogr. 19, 466-473. doi: 10.1002/bmc.515

Porcheron, G., Habib, R., Houle, S., Caza, M., Lépine, F., Daigle, F., et al. (2014). The small RNA RyhB contributes to siderophore production and virulence of uropathogenic Escherichia coli. Infect. Immun. 82, 5056-5068. doi: 10.1128/IAI.02287-14

Psychogios, N., Hau, D. D., Peng, J., Guo, A. C., Mandal, R., Bouatra, S., et al. (2011). The human serum metabolome. PLoS ONE 6:e16957. doi: 10.1371 /journal.pone.0016957

Rang, C. U., Licht, T. R., Midtvedt, T., Conway, P. L., Chao, L., Krogfelt, K. A., et al. (1999). Estimation of growth rates of Escherichia coli BJ4 in streptomycintreated and previously germfree mice by in situ rRNA hybridization. Clin. Diagn. Lab. Immunol. 6, 434-436.

Rios, L. Y., Gonthier, M.-P., Remesy, C., Mila, I., Lapierre, C., Lazarus, S. A., et al. (2003). Chocolate intake incrases urinary excretion of polyphenotderived phenolic acids in healthy human subjects. Am. J. Clin. Nutr. 77, 912-918.

Roesch, P. L., Redford, P., Batchelet, S., Moritz, R. L., Pellett, S., Haugen, B. J., et al. (2003). Uropathogenic Escherichia coli use D-serine deaminise to modulate infection of the murine urinary tract. Mol. Microbiol. 49, 55-67. doi: 10.1046/j.1365-2958.2003.03543.x

Romeo, T. (1998). Global regulation by the small RNA-binding protein CsrA and the non-coding RNA molecule CsrB. Mol. Microbiol. 29, 1321-1330. doi: 10.1046/j.1365-2958.1998.01021.x

Rosen, D. A., Hooton, T. M., Stamm, W. E., Humphrey, P. A., and Hultgren, S. J. (2007). Detection of intracellular bacterial communities in human urinary tract infection. PLoS Med. 4, 1949-1958. doi: 10.1371/journal.pmed. 0040329

Samant, S., Lee, H., Ghassemi, M., Chen, J., Cook, J. L., Mankin, A. S., et al. (2008). Nucleotide biosynthesis is critical for growth of bacteria in human blood. PLoS Pathog. 4:e37. doi: 10.1371/journal.ppat.0040037

Saude, E. J., Adamko, D., Rowe, B. H., Marrie, T., and Sykes, B. D. (2007). Variations of metabolites in normal human urine. Metabolomics 3, 439-451. doi: 10.1007/s11306-007-0091-1

Schulz, W. A. (2011). Uropathogenic bacteria leave a mark. Lab. Invest. 91, 816-818. doi: 10.1038/labinvest.2011.51

Severi, E., Hood, D. W., and Thomas, G. H. (2007). Sialic acid utilization by bacterial pathogens. Microbiology 153, 2817-2822. doi: 10.1099/mic.0.2007/009480-0
Shaffer, C. L., Zhang, E. W., Dudley, A. G., Dixon, B. R. E. A., Guckes, K. R., Breland, E. J., et al. (2017). Purine biosynthesis metabolically constrains intracellular survival of Uropathogenic Escherichia coli. Infect. Immun. 85, e00471-e00416. doi: 10.1128/iai.00471-16

Shields-Cutler, R. R., Crowley, J. R., Hung, C. S., Stapleton, A. E., Aldrich, C. C., Marschall, J., et al. (2015). Human urinary composition controls antibacterial activity of siderocalin. J. Biol. Chem. 290, 15949-15960. doi: 10.1074/jbc.M115.645812

Sjölin, J., Hjort, G., Friman, G., and Hambraeus, L. (1987). Urinary excretion of 1-methylhistidine: a qualitative indicator of exogenous 3-methylhistidine and intake of meats from various sources. Metabolism 36, 1175-1184. doi: 10.1016/0026-0495(87)90245-9

Snyder, J. A., Haugen, B. J., Buckles, E. L., Lockatell, V., Johnson, D. E., Michael, S., et al. (2004). Transcriptome of Uropathogenic Escherichia coli during Urinary Tract Infection. Infect. Immun. 72, 6373-6381. doi: 10.1128/IAI.72.11.6373-6381.2004

Stamey, T. A., and Mihara, G. (1980). Observations on the growth of urethral and vaginal bacteria in sterile urine. J. Urol. 124, 461-463.

Stamm, W. E., and Norrby, S. R. (2001). Urinary tract infections: disease panorama and challenges. J. Infect. Dis. 183, S1-S4. doi: 10.1086/318850

$\mathrm{Su}$, Q., Guan, T., He, Y., and Lv, H. (2016a). Siderophore biosynthesis governs the virulence of Uropathogenic Escherichia coli by coordinately modulating the differential metabolism. J. Proteome Res. 15, 1323-1332. doi: 10.1021/acs.jproteome.6b00061

Su, Q., Guan, T., and Lv, H. (2016b). Siderophore biosynthesis coordinately modulated the virulence-associated interactive metabolome of uropathogenic Escherichia coli and human urine. Sci. Rep. 6:24099. doi: 10.1038/srep 24099

Subashchandrabose, S., Hazen, T. H., Brumbaugh, A. R., Himpsl, S. D., Smith, S. N., Ernst, R. D., et al. (2014). Host-specific induction of Escherichia coli fitness genes during human urinary tract infection. Proc. Natl. Acad. Sci. U.S.A. 111, 18327-18332. doi: 10.1073/pnas.1415959112

Suzuki, K., Wany, X., Weilbacher, T., Pernestig, A. K., Melefors, O., Georgellis, D., et al. (2002). Regulatory circuitry of the CsrA/CsrB and BarA/UvrY systems of Escherichia coli. J. Bacteriol. 188, 5130-5140. doi: 10.1128/jb.184.18.5130-5140.2002

Sweeney, N. J., Laux, D. C., and Cohen, P. S. (1996). Escherichia coli F-18 and E. coli K-12 eda mutants do not colonize the streptomycin-treated mouse large intestine. Infect. Immun. 64, 3504-3511.

Takeda, I., Stretch, C., Barnaby, P., Bhatnager, K., Rankin, K., Fub, H., et al. (2009). Understanding the human salivary metabolome. NMR Biomed. 22, 577-584. doi: $10.1002 / \mathrm{nbm} .1369$

Taylor, E. H., Fink, L. M., and Pappas, A. A. (1989). Reproducibility of creatine kinase isoenzyme electrophoresis. Clin. Chem. 35:710.

Tomenius, H., Pernestig, A.-K., Jonas, K., Georgellis, D., Möllby, R., Normark, S., et al. (2006). The Escherichia coli BarA-UvrY two-component system is a virulence determinant in the urinary tract. BMC Microbiol. 6:27. doi: $10.1186 / 1471-2180-6-27$

Torres, A. G., Redford, P., Welch, R. A., and Payne, S. M. (2001). TonB-dependent systems of Uropathogenic Escherichia coli : aerobactin and heme transport and TonB are required for virulence in the mouse. Infect. Immun. 69, 6179-6185. doi: 10.1128/IAI.69.10.6179-6185.2001

Vejborg, R. M., De Evgrafov, M. R., Phan, M. D., Totsika, M., Schembri, M. A., and Hancock, V. (2012). Identification of genes important for urine growth of asymptomatic bacteriuria Escherichia coli. Infect. Immun. 80, 3179-3188. doi: 10.1128/IAI.00473-12

Weber, H., Polen, T., Heuveling, J., Wendisch, V. F., and Hengge, R. (2005). Genome-Wide analysis of the general stress response network in Escherichia coli: $\sigma$ S -Dependent genes, promoters, and Sigma factor selectivity. J. Bacteriol. 187, 1591-1603. doi: 10.1128/JB.187.5.1591-16 03.2005

Weilbacher, T., Suzuki, K., Dubey, A. K., Wang, X., Gudapaty, S., Morozov, I., et al. (2003). A novel sRNA component of the carbon storage regulatory system of Escherichia coli. Mol. Microbiol. 48, 657-670. doi: 10.1046/j.1365-2958.2003.03459.x

Wieser, A., Romann, E., Magistro, G., Hoffmann, C., Nörenberg, D., Weinert, K., et al. (2010). A multiepitope subunit vaccine conveys protection against 
extraintestinal pathogenic Escherichia coli in mice. Infect. Immun. 78, 3432-3442. doi: 10.1128/IAI.00174-10

Wiles, T. J., Kulesus, R. R., and Mulvey, M. A. (2008). Origins and virulence mechanisms of uropathogenic Escherichia coli. Exp. Mol. Pathol. 85, 11-19. doi: 10.1016/j.yexmp.2008.03.007

Wishart, D. S. (2016). Applications of metabolomics in drug discovery and development. Nat. Rev. Drug Discov. 15, 473-484. doi: 10.1038/nrd. 2016.32

Yan, L., Nie, W., and Lv, H. (2015). Metabolic phenotyping of the Yersinia highpathogenicity island that regulates central carbon metabolism. Analyst 140, 3356-3361. doi: 10.1039/C4AN02223H
Conflict of Interest Statement: The authors declare that the research was conducted in the absence of any commercial or financial relationships that could be construed as a potential conflict of interest.

Copyright (๑) 2017 Mann, Mediati, Duggin, Harry and Bottomley. This is an openaccess article distributed under the terms of the Creative Commons Attribution License (CC BY). The use, distribution or reproduction in other forums is permitted, provided the original author(s) or licensor are credited and that the original publication in this journal is cited, in accordance with accepted academic practice. No use, distribution or reproduction is permitted which does not comply with these terms. 